\title{
Neural Mechanisms Underlying Adaptive Actions after Slips
}

\author{
Josep Marco-Pallarés ${ }^{1}$, Estela Camara ${ }^{1,2}$, Thomas F. Münte ${ }^{1}$, \\ and Antoni Rodríguez-Fornells ${ }^{2,3}$
}

\begin{abstract}
An increase in cognitive control has been systematically observed in responses produced immediately after the commission of an error. Such responses show a delay in reaction time (post-error slowing) and an increase in accuracy. To characterize the neurophysiological mechanism involved in the adaptation of cognitive control, we examined oscillatory electrical brain activity by electroencephalogram and its corresponding neural network by event-related functional magnetic resonance imaging in three experiments. We identified a new oscillatory theta-beta component related to the degree of post-error slowing in the
\end{abstract}

\section{INTRODUCTION}

To err is certainly human. Learning from our errors entails, at the simplest level, the correction (where possible) of the error and the instigation of actions to prevent or remedy similar errors in the future. By applying electrophysiological and hemodynamic measures, this study seeks to define the neurophysiological dynamics and mechanisms underlying such adaptive processes.

The production of erroneous responses in reaction time (RT) tasks is typically followed by a delay in the production of correct responses in subsequent trials (Rabbitt, 1966). Such performance changes have often been attributed to between-trial executive control adjustments. As extended processing is allowed in those trials that follow an error, more time can be devoted to stimulus identification and response selection processes, which avoids the commission of premature or impulsive responses on the basis of insufficient evidence. These trial-by-trial adaptations enhance the probability of producing correct responses in trials following erroneous responses. Post-error slowing occurs independently of the characteristics of the previous trial, which contrasts with the other well-known between-trial adaptation process, conflict adaptation, which appears subsequent to

\footnotetext{
${ }^{1}$ Otto von Guericke University, Magdeburg, Germany, ${ }^{2}$ University of Barcelona, ${ }^{3}$ Institució Catalana de Recerca i Estudis Avançats (ICREA)
}

correct responses following an erroneous trial. Additionally, we found that the activity of the right dorsolateral prefrontal cortex, the right inferior frontal cortex, and the right superior frontal cortex was correlated with the degree of caution shown in the trial following the commission of an error. Given the overlap between this brain network and the regions activated by the need to inhibit motor responses in a stop-signal manipulation, we conclude that the increase in cognitive control observed after the commission of an error is implemented through the participation of an inhibitory mechanism.

high-conflict trials (Kerns et al., 2004; Gratton, Coles, \& Donchin, 1992; but see Wendt, Heldmann, Munte, \& Kluwe, 2007; Mayr, Awh, \& Laurey, 2003).

Interestingly, post-error slowing seems to be automatically triggered following the commission of an error and it appears to occur independently of awareness. In an elegant series of studies in which participants were required to signal whether they had committed an error or not, the post-error slowing effect was observed even in those trials that were not consciously registered and signaled (Rabbitt, 1968, 1990, 2002). This result clearly shows the automatic and involuntary character of posterror slowing. Furthermore, psychopharmacological agents that either increase or disrupt the error detection process had no effect on post-error slowing (Riba, RodriguezFornells, Morte, Munte, \& Barbanoj, 2005; Riba, RodriguezFornells, Munte, \& Barbanoj, 2005). In this study, a component of the event-related brain potential (ERP), the error-related negativity (ERN), was used to assess the activity of the error detection system (Gehring, Goss, Coles, Meyer, \& Donchin, 1993; Falkenstein, Hohnsbein, Hoormann, \& Blanke, 1990). Finally, (Rabbitt, 1966) showed that the degree of post-error slowing was unrelated to either the RT of the erroneous responses or to correction times. These studies therefore suggest that post-error adaptive mechanisms operate, at least partially, independently of error detection and correction processes (Rodriguez-Fornells, Kurzbuch, \& Munte, 2002).

Despite the robustness and importance of post-error slowing, the cognitive control and neural mechanisms 
that sustain trial-by-trial adaptations have remained largely unknown in spite of some explanatory attempts. The earliest interpretations postulated that post-error slowing reflects the activation of an "error-sensitive control process" (Laming, 1979; Burns, 1971; Rabbitt, 1966), whereby the system adopts a more conservative or cautious response bias following the commission of an error, producing slower responses that are more likely to be correct. Two specific hypotheses have been proposed to further specify this strategy. The inbibitory account (Ridderinkhof, 2002) holds that, after the commission of an error, an increase in the strength of selective suppression or inhibition on subsequent trials is observed. This hypothesis is based on the observation that the amount of interference of irrelevant dimensions in posterror trials is less than for trials following a correct response, implying increased inhibitory control after errors (Ridderinkhof, 2002). Alternatively, the conflict monitoring account (Botvinick, Braver, Barch, Carter, \& Cohen, 2001) suggests that after the detection of conflict in an erroneous trial, the activation of the conflict monitoring system is increased (reflected in the activation of the anterior cingulate cortex, ACC), triggering cognitive control (most probably through the recruitment of the prefrontal cortex $[\mathrm{PFC}]$ ) and reducing excitatory input to the motorresponse level. Therefore, if conflict is detected, cognitive control increases and response activation is reduced, ensuring that the trial immediately following an error will show slower RTs, and thus, more accurate responses. Thus, considering this model, cognitive control is considered a top-down signal that biases processing and minimizes the possibility of conflict in a context-dependent fashion (Egner \& Hirsch, 2005a). In agreement with these findings, functional magnetic resonance imaging (fMRI) studies have demonstrated that the activation of ACC (Kerns et al., 2004; Garavan, Ross, Murphy, Roche, \& Stein, 2002) and the presupplementary motor area [preSMA] (Klein et al., 2007) in erroneous trials is positively related to post-error slowing. Furthermore, the amplitude of the ERN has been found to be related to the amount of post-error slowing (Debener et al., 2005; Gehring et al., 1993). However, this interesting finding has not always been replicated (Gehring \& Fencsik, 2001; Nieuwenhuis, Ridderinkhof, Blom, Band, \& Kok, 2001).

Both the inbibitory and the conflict monitoring accounts propose that cognitive control is engaged after the commission of an error, being responsible for the post-error slowing phenomena. Despite their obvious differences, both accounts propose that the activation of the motor system is biased, either by direct response suppression (inhibition) or by reducing the amount of activation of the response channels. For example, in simulations based on the conflict monitoring account (Simulation 2C, Botvinick et al., 2001), the conflict-control loop directly affects the degree of response priming of the system, not only after the commission of errors (which is a high-conflict situation) but also after any type of trial.
Thus, the conflict-control loop acts as a system that regulates the balance between speed and accuracy. The degree of conflict associated with a given response will produce a corresponding change in the degree of priming at the motor level. Low-conflict trials will move the system to a more risky state, elevating response activation on the next trials, speeding up reactions, and increasing chances for erroneous responses. In contrast, high-conflict trials will diminish response activation and, therefore, slower RTs and a more cautious behavior will be observed on the following trials.

By contrast, Ridderinkhof (2002) stressed that trial-bytrial micro-adjustments of behavior, as evidenced by posterror slowing or by sequential effects (i.e., RT interference is reduced on trials that are preceded by noncompatible than compatible trials), involve selective suppression, namely, inhibition, of fast processing (via a direct route in Ridderinkhof's terms) to give more time for processing via a slower "deliberate route." Thus, the key feature of Ridderinkhof's model is inhibition of response activation.

The goal of the present study is to further investigate the underlying neurophysiological mechanisms involved in post-error slowing. In the first experiment, using ERPs and time-frequency analysis, we characterize a new component of oscillatory brain activity in the theta and beta ranges associated with post-error slowing. In the second experiment, we used fMRI to demonstrate that post-error slowing modulates the activity of those brain regions predicted by the inhibitory account. A further ERP experiment was conducted to show that the previously isolated theta-beta oscillatory component is associated to the inhibition of motor commands.

\section{METHODS}

All procedures were approved by the institutional review board of the University of Magdeburg.

\section{Experiment 1}

\section{Participants}

Twenty-four right-handed volunteers participated in the study (15 women; $27 \pm 4$ years) after signing an informed consent form in accordance with the Declaration of Helsinki. They were paid for their participation.

\section{Task and Stimulus Materials}

A modified version of the Eriksen flanker task was used. Participants were instructed to focus their attention on the center of a screen and to respond to the central letter in five-letter arrays with either their right (letter $\mathrm{H}$ ) or left (letter S) hand. The four letters flanking the central (target) letter were either compatible $(\mathrm{HHHHH}$, 
SSSSS), that is, requiring the same response as the target letter, or incompatible (HHSHH, SSHSS), priming the erroneous response. Each stimulus array subtended $\sim 2.5^{\circ}$ of visual angle in width, and a fixation line was presented in the middle of the computer monitor just below the target letter in the array. In addition, in half of the trials, the target letter appeared degraded by removing $\sim 70 \%$ of the pixels (the flankers were maintained nondegraded). The duration of the stimuli was $100 \mathrm{msec}$, and the stimulus onset asynchrony (SOA) between two successive stimuli was 900 msec. Letter-hand assignments were counterbalanced between participants. To increase the number of errors, $60 \%$ of the stimuli were incompatible. Participants were encouraged to correct their errors as fast as possible by pressing the correct button. Participants performed 24 to 26 blocks of 200 stimuli and were allowed 2 min rest between blocks.

\section{EEG Recording and Data Analysis}

Electroencephalogram (EEG; bandpass $=0.01-70 \mathrm{~Hz}$; digitization rate $=250 \mathrm{~Hz}$ ) was recorded from 29 tin electrodes in an elastic cap including all standard positions of the 10-20 system. The EEG signals were rereferenced to the mean activity at the mastoid electrodes. Vertical and horizontal eye electrodes were recorded by bipolar montages and used for artifact rejection purposes.

Epochs capturing the activity evoked by three consecutive stimuli (length $=2800 \mathrm{msec}$, starting $100 \mathrm{msec}$ prior to the first stimulus and ending $900 \mathrm{msec}$ after the onset of the third) were extracted from the EEG. The presentation of the first stimulus is referred to as S1, the second as S2, and the third as S3; the responses to the respective stimuli are recorded as R1, R2, and R3. Two different epochs were selected. The first epoch, henceforth CCC, contained the electrical activity associated to three consecutive correct trials, whereas the second epoch comprised the EEG signal from a correct-error-correct sequence (henceforth CEC). Epochs in which EEG or electrooculogram activity exceeded $\pm 50 \mu \mathrm{V}$ were rejected from further analysis.

Event-related brain potentials. ERPs were obtained separately for CCC and CEC epochs. Stimulus-locked (S1, S2, and S3) and response-locked (R1, R2, and R3) epochs were computed separately. The baseline in all cases was established between -160 and 0 msec before the appearance of the S2, as proposed by Picton et al. (2000). However, virtually identical results were obtained when the baseline was set prior to responses (i.e., R2). For statistical analysis, mean amplitude measures in both epochs (CCC and CEC) and for the three midline electrode locations (Fz, Cz, Pz) were obtained and entered into the corresponding analysis of variance. The Greenhouse-Geisser epsilon correction was applied when necessary.
Time-frequency analysis. In addition to ERPs, timefrequency analysis was performed by convoluting singletrial data with a complex Morlet wavelet:

$$
w\left(t, f_{0}\right)=\left(2 \pi \sigma_{t}^{2}\right)^{-1 / 2} e^{\frac{-t^{2}}{2 \sigma_{t}^{2}}} e^{2 i \pi f_{0} t}
$$

where the relation $f_{0} / \sigma_{f}$ (where $\sigma_{f}=1 /\left(2 \pi \sigma_{t}\right)$ ) was set to 6.7 (Tallon-Baudry, Bertrand, Delpuech, \& Permier, 1997). The frequencies studied ranged from 1 to $40 \mathrm{~Hz}$, with a linear increase of $1 \mathrm{~Hz}$. The time-varying energy (square of the convolution between wavelet and signal) was computed for each trial and was averaged separately for each subject. Then, the percentage change with respect to a baseline set at -160 to 0 msec before S2 was computed for each subject. Time-frequency contents were averaged stimulus- and response-locked (R1, R2, R3). To study significant increases/decreases in power between the different conditions, Mann-Wilcoxon ranksum tests were performed for all electrodes, frequencies, and time points. Differences were only considered relevant if significant for more than 100 consecutive milliseconds. In addition, mean increase/decrease in power in both epochs (CCC and CEC) and for the three midline electrode locations $(\mathrm{Fz}, \mathrm{Cz}, \mathrm{Pz})$ were obtained and entered into analyses of variance. The Greenhouse-Geisser epsilon correction was applied when necessary.

\section{Experiment 2}

\section{Participants}

Ten different right-handed paid volunteers (mean age 23 years; 7 women) gave their written informed consent.

\section{Task and Stimulus Materials}

A similar task was used as in the previous experiment, but with two changes. First, the SOA between two successive stimuli in this experiment was a random interval between 1750 and $2250 \mathrm{msec}$ (rectangular distribution). Second, we included a variant of the stop-signal paradigm (Stuphorn, Taylor, \& Schall, 2000; Logan, Cowan, \& Davis, 1984). In $25 \%$ of the trials, a stop-signal was presented. This comprised a red square surrounding the central target letter. Volunteers were instructed to inhibit their own response as soon as the red square appeared. The stop signal was presented using two different delays (equal probability for each delay). The latency of these delays from the imperative stimuli was established considering the individual subject's mean RT obtained in the training phase (easy to inhibit delay, mean RT minus $225 \mathrm{msec}$, and hard to inhibit delay, mean RT minus $75 \mathrm{msec}$ ).

Prior to the experiment and outside of the scanner, participants were initially trained with 400 trials to reach a baseline RT that was used as a starting point to fix the final individual RT deadline. After this baseline period, a 
series of 40 trials were administered and the participants received feedback about their performance. The goal of this procedure was to aim for an RT that would yield approximately $10 \%$ to $15 \%$ erroneous responses.

Eight runs of 200 trials and approximately $5 \mathrm{~min}$ duration separated by short rest periods were administered. Participants were encouraged to correct the erroneous responses as fast as possible. Those participants who did not commit a sufficient number of corrected errors performed an additional run. The stimuli were projected onto a mirror in direct view of the reclining volunteer, and responses were given using response boxes.

\section{MRI Scanning Methods}

MRI data were collected using a GE Medical Systems 1.5Tesla Signa Neurovascular MR scanner with standard quadrature head coil. Visual images were back-projected onto a screen using an light-emitting diode projector and participants viewed the images through a mirror on the head coil. Magnet-compatible response buttons were used. Conventional high-resolution structural images (radio-frequency-spoiled GRASS sequence, 60 slice sagittal, $2.8 \mathrm{~mm}$ thickness) were followed by functional images sensitive to blood oxygenation level-dependent contrast (echo-planar $\mathrm{T}_{2}{ }^{*}$-weighted gradient-echo sequence, $\mathrm{TR}=2000 \mathrm{msec}, \mathrm{TE}=40 \mathrm{msec}$, flip $=90^{\circ}$ ). Each functional run consisted of 215 sequential wholebrain volumes comprising 23 axial slices aligned to the plane intersecting the anterior and posterior commissures, $3.125 \mathrm{~mm}$ in-plane resolution, $6 \mathrm{~mm}$ thickness, $1 \mathrm{~mm}$ gap between slices, positioned to cover all but the most superior region of the brain and the cerebellum. Volumes were acquired continuously and the first four volumes were discarded to allow for T1 equilibration effects.

\section{Preprocessing}

Data were analyzed using standard procedures implemented in the Statistical Parametric Mapping software (SPM2, www.fil.ion.ucl.ac.uk/spm). The preprocessing included slice-timing, realignment, normalization, and smoothing. First, functional volumes were phase-shifted in time with reference to the first slice to minimize purely acquisition-dependent signal variations across slices. Head movement artifacts were corrected based on an affine rigid-body transformation, where the reference volume was the first image of the first run (e.g., Friston, Williams, Howard, Frackowiak, \& Turner, 1996). Functional data were then averaged and the mean functional image was normalized to a standard stereotactic space using the EPI-derived Montreal Neurological Institute (MNI) template (ICBM 152) provided by SPM2. After an initial 12-parameter affine transformation, an iterative nonlinear normalization was applied using discrete cosine basis functions by which brain warps are expanded in
SPM2 (Ashburner \& Friston, 1999). Resulting normalization parameters derived for the mean image were applied to the whole functional set. Finally, functional EPI volumes were resampled into $4-\mathrm{mm}^{3}$ voxels and then spatially smoothed with an 8-mm full-width half-maximum isotropic Gaussian kernel to minimize effects of intersubject anatomical differences.

\section{Data Analysis}

The statistical evaluation was based on a least-square estimation using the general linear model by modeling the different conditions with a regressor waveform convolved with a canonical hemodynamic response function (Friston et al., 1998). First, an event-related design matrix was created including the conditions of interest: correct (C) and erroneous (E; corrected choice errors only) responses, inhibited stop trials and noninhibited stop trials. We used this analysis to define the brain regions activated in these conditions. In a second analysis, we created a new design matrix that replaced the standard erroneous and correct trials by the critical sequences of interest: correct-correct trials (CC), error-correct trials (EC) sequences. The onset was defined for the second member of the sequence (always the correct response). Finally, we also included stimulus-response compatibility as a factor in the definition of the CC sequences. This factor was included in order to evaluate possible postconflict interactions. These sequences were defined as follows: incongruent trials preceded by an incongruent trial and incongruent trials preceded by a congruent one; congruent trials preceded by a congruent trial and congruent trials preceded by an incongruent one; and, by contrast, incongruent trials preceded by a congruent trial and incongruent trials preceded by congruent one.

The data were high-pass filtered (to a maximum of 1/ $90 \mathrm{~Hz}$ ), and serial autocorrelations were estimated using an autoregressive model $[\mathrm{AR}(1)]$. Resulting estimates were used for nonsphericity correction during the model estimation. Confounding effects in global mean were removed by proportional scaling, and signal-correlated motion effects were minimized by including the estimated movement parameters. The individual contrast images were entered into a second-level analysis using a one-sample $t$ test employing a random effects analysis within the general linear model. Unless mentioned otherwise, contrasts were thresholded at $p<.001$, and only clusters with a significant $p<.001$, corrected for multiple comparisons, were reported and interpreted (Worsley \& Friston, 1995). The maxima of suprathreshold regions were localized by rendering them onto the mean volunteers' normalized T1 structural images on the MNI reference brain (Cocosco, Kollokian, Kwan, \& Evan, 1997). Maxima and all coordinates are reported in MNI coordinates, as used by SPM and labeled in line with the Talairach atlas. 


\section{ROI Analysis}

Regions of interest (ROI) used for within-region comparison were created by selecting voxels with overlap between the group full second-level analysis in the EC versus CC contrast. The right dorsolateral prefrontal cortex (DLPFC) was defined by thresholding to $p<.001$ (uncorrected). Data-averaged time series were collected from intersecting voxels and were baseline corrected for every subject. The signal intensity of the voxels in each ROI was averaged for each subject, and the peak activation was compared within-subjects between the EC and CC conditions.

\section{Experiment 3}

\section{Participants}

Twenty-four different right-handed paid volunteers participated in the study (18 women; $22.6 \pm 4.1$ years).

\section{Task and Stimulus Materials}

A modified version of the Eriksen flanker task was used. Participants were instructed to focus their attention on the center of a screen and to respond by indicating in the same direction as that shown by the arrow presented at the center of the array (five arrows). The four arrows flanking the central (target) arrow were either compatible (same direction as the one in the center) or incompatible (opposite direction), priming the erroneous response. Each stimulus array subtended $\sim 2.5^{\circ}$ of visual angle in width, and a fixation line was presented in the middle of the computer monitor just below the target letter in the array. Duration of the stimuli was $300 \mathrm{msec}$, and the SOA between two successive stimuli was $900 \mathrm{msec}$. In order to increase the number of erroneous responses, $60 \%$ of the stimuli presented were incompatible. Participants were encouraged to correct their errors by pressing the correct button. In addition, in $17 \%$ of trials, a stop signal was presented. In these trials, the center arrow turned red and participants were requested to inhibit their response.

In order to obtain an equal number of inhibited and noninhibited responses, we used a staircase tracking algorithm (Band \& van Boxtel, 1999): If the participant is able to inhibit successfully a stop trial, then inhibition is made more difficult on the subsequent trial increasing the stop-signal delay, that is, separating the imperative stimulus and the stop signal. If the participant is not able to inhibit the response, then inhibition is made easier reducing the stop-signal delay. In this particular design, the stop-signal delay was set initially at $140 \mathrm{msec}$. After a successful inhibition, $10 \mathrm{msec}$ were subtracted from the stop-signal delay. After unsuccessful inhibitions, the stopsignal delay was increased by $10 \mathrm{msec}$. Participants performed eight blocks of 240 stimuli each and were allowed 2 min rest between blocks.

\section{EEG Recording and Analysis}

Recording and artifact rejection characteristics were similar to Experiment 1.

The analysis focuses on the stop trials, which were separated into successful and unsuccessful trials. ERPs were computed from -100 to $1000 \mathrm{msec}$ after the stop signal. ERPs were obtained separately for inhibited and noninhibited conditions. Statistical and time-frequency analyses were performed as explained in Experiment 1.

\section{RESULTS}

\section{Experiment 1}

\section{Behavioral Results}

The error rate was $19 \pm 5 \%$, with erroneous responses being significantly faster than correct responses [324 \pm 30 msec vs. $361 \pm 29 \mathrm{msec}, t(23)=15.7, p<.001]$. Errors were corrected in $93 \pm 7 \%$ of the cases with a mean correction latency of $206 \pm 35 \mathrm{msec}$. The mean percentage of correct responses after the commission of an error was higher than after a preceding correct answer [ $82 \pm$ $6 \%$ vs. $80 \pm 6 \%, t(23)=2.46, p<.05]$. Also, the mean RT for correct responses after an error (377 $\pm 32 \mathrm{msec})$ was significantly slower than that for correct responses after a correct response $[356 \pm 30 \mathrm{msec}, t(23)=15.7$, $p<.001]$. Thus, post-error slowing amounted to $21 \pm$ $21 \mathrm{msec}$.

\section{Event-related Brain Potentials}

Stimulus-locked ERPs for CCC and CEC stimulus triads are depicted in Figure 1A. During the first correct response in each triad (S1), no differences were observed between conditions and the corresponding ERP responses showed a perfect overlap. After the presentation of the second stimuli (S2), both waveforms began to diverge at about $400 \mathrm{msec}$ due to the error in the second response. These differences were also apparent in the R2 response-locked ERPs (see Figure 1B) showing a typical fronto-central ERN component, peaking at about $80 \mathrm{msec}$ after the commission of an error (for scalp distribution, see Figure 1C). The ERN was followed by a centro-parietal positive component, known as error positivity (Pe; for scalp distribution, see Figure 1D). The corresponding statistical analysis showed that correct and erroneous trials differed between 0 and $100 \mathrm{msec}$ (ERN component) after the response (R2) [Condition: $F(1,23)=115.9, p<.001$; Condition $\times$ Electrode: $F(2,46)=22.38, p<.001]$. For the Pe component (mean amplitude 200-500 msec), correct and erroneous responses also differed significantly [Condition, $F(1,23)=83.0, p<.001$; Condition $\times$ Electrode: $F(2,46)=22.38$; Condition $\times$ Electrode: $F(2$, 46) $=65.50, p<.001]$ 
Figure 1. Event-related potentials of correct and error responses. (A) Stimulus-locked ERPs at frontal (Fz) and central locations $(\mathrm{Cz})$ for correctcorrect-correct (CCC, solid line) and correct-error-correct (CEC, dashed line) epochs locked to the appearance of the first stimulus (labeled as S1). Depicted also is the onset of the next two stimuli (S2 and S3, $900 \mathrm{msec}$ SOA). Notice the perfect match between the ERP responses to the first correct trials, and the difference that appeared after the second trial (S2), where the erroneous response is produced (unfiltered data). (B) Response-locked ERPs locked to the production of the second response (R2) which was a correct (CC) or erroneous (EC) response. The two components associated with the commission of errors-the ERN and the error positivity $(\mathrm{Pe})$ - can be observed. (C) Isovoltage maps of the difference waveform (R2 locked) computed by subtracting CEC versus CCC at the time range indicated after R2. Note the fronto-central negativity characterizing the ERN and the centro-parietal positivity of the Pe.

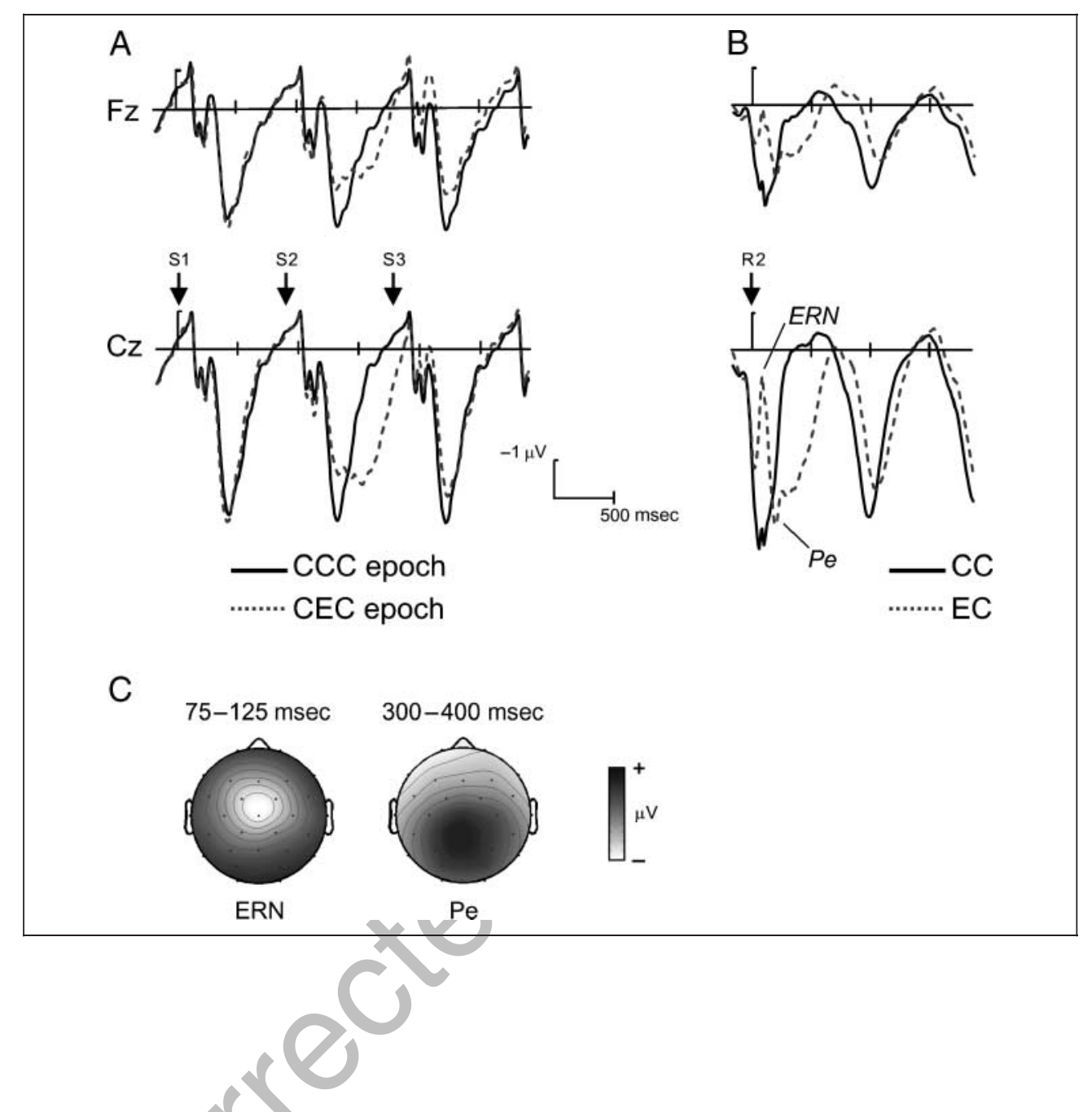

\section{Time-frequency Analysis}

Figure 2 shows the time-frequency analysis time-locked to the onset of the second stimulus (S2). A significant increase in the theta band power $(4-8 \mathrm{~Hz})$ was found after 100 to $900 \mathrm{msec}$ in the case of the erroneous trials, which was considerably stronger than the theta-response in the correct trials (see Figure $2 \mathrm{~A}-\mathrm{B}$, and statistical analysis at Figure 2D). This theta component, associated to erroneous processing, has been identified in several previous studies (Gehring \& Willoughby, 2004; Yeung, Botvinick, \& Cohen, 2004; Luu, Tucker, Derryberry, Reed, \& Poulsen, 2003) and differed significantly between error and correct trials [300-600 msec; Condition: $F(1,23)=$ $47.2, p<.001$; Condition $\times$ Electrode: $F(2,46)=23.2$, $p<.001$; see scalp distribution at Figure 2C). Moreover, an increase in the beta power $(20-25 \mathrm{~Hz})$ was found in the time range coinciding with the occurrence of the third stimuli (S3) (in between 900 and 1100 msec after S2), which was more pronounced in the erroneous trials (see Figure $2 \mathrm{C}$ and $\mathrm{D})$. A significant condition effect $[F(1$, $23)=21.1, p<.001]$ and interaction between condition and midline electrode was found in this time range $[F(2$,
$46)=5.2, p<.05]$, the latter reflecting its central distribution (Figure 2C).

Both effects were also observed when the analysis was performed time-locked to the second response (R2): The increase in power in the theta band for incorrect responses had an onset slightly before R2 and extended until 400 msec after R2 in the erroneous trials (see Figure 3). There was a significant increase in the theta band (4-8 Hz) between 0 and $100 \mathrm{msec}$, which is associated to the ERN [Condition: $F(1,23)=56.2, p<.001$; Condition $\times$ Electrode: $F(2,46)=23.1, p<.001$; a significant difference was encountered at all midline electrodes, $t(23)>4, p<.005]$. There were also significant differences between error and correct trials in the $200-500 \mathrm{msec}$ time window [Condition: $F(1,23)=32.8, p<.001$; Condition $\times$ Electrode: $F(2,46)=22.8, p<.001]$. An increase in beta power was detected from 600 to 800 msec after R2 (see Figure 3A-C, and statistical analysis in Figure 3D). A significant interaction between Condition $\times$ Electrode was found $[20-25 \mathrm{~Hz}$, time window $600-800 \mathrm{msec}$; Condition: $F(1,23)=30.9, p<.001$; Condition $\times$ Electrode: $F(2,46)=10.1, p<.005$ ], reflecting a fronto-central distribution (see Figure 3 ). 


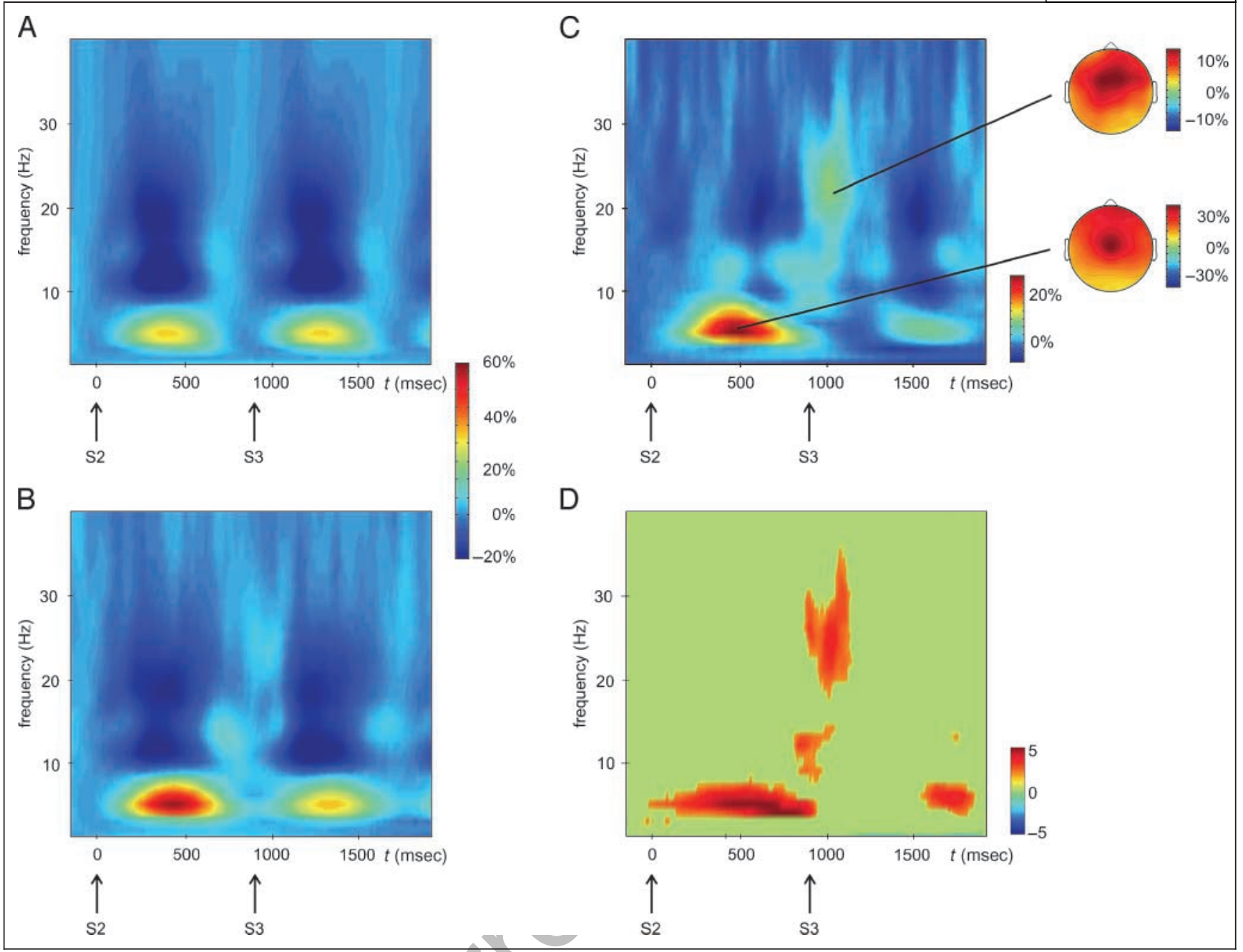

Figure 2. Time-frequency plots for S2 locked data. (A, B) Change of power relative to baseline ( -160 to 0 msec prior to S2) for the correct-correct-correct (CCC) condition (A) and correct-error-correct condition (CEC) (B), from 1 to $40 \mathrm{~Hz}$ at Fz location. Depicted also is the onset of stimuli S2 and S3 (900 msec SOA). (C) Differences in the power change between CEC and CCC conditions at Fz electrode. Note the central topography of the theta component (in the R2 time range) and the fronto-central topography of the beta component appearing in the S3 time range. (D) Point-by-point Mann-Wilcoxon tests between CEC and CCC conditions at Fz. Only significant values $(p<.05)$ that lasted a minimum of 100 msec are represented.

To determine whether the power of beta activity following the erroneous response was related to the power of theta activity associated with the ERN and Pe components, we sorted single trials for each participant into three bins according beta power at Fz (20-25 Hz; 600-800 msec after R2): high, medium, and low beta activity. In these groups of trials, the power of theta activity (4-8 Hz) was computed in two different time windows: the activity associated with the ERN (0-100 msec after R2) and that associated with the Pe component (200-500 msec after R2). High beta trials had higher theta power within the early "ERN" time window (0$100 \mathrm{msec})$ in comparison with low beta trials $[t(23)=$ $2.55, p<.05]$. Moreover, a significant linear increase of theta power was found in these three groups of trials $[F(1,23)=6.51, p<.05$; see Figure 4]. A similar relation was not found in the following time window
(200-500 msec, linear trend $p>.1$ ). This analysis demonstrates that the beta oscillatory component is coupled with the power of theta underlying the ERN but not the Pe component.

To examine the relationship between this beta oscillatory activity and post-error slowing in the correct trials following erroneous responses, we classified these trials according to their RT to R3 into three groups: fast, medium, and slow responses. The beta activity differed between these groups of trials $[F(2,46)=15.6, p<.001]$, with a significant linear increase in the beta activity with RT $[F(1,23)=17.7, p<.001$; see Figure $5 \mathrm{~A}]$. Moreover, a significant correlation between R3 RT and beta activity was found in the group of slow trials $[r=.418, F(1$, $22)=4.6, p<.05]$, but not in the medium and fast response trials $(r<.3, p>.1)$ (see Figure $5 \mathrm{~B})$. When combining the three groups of trials, a highly significant 


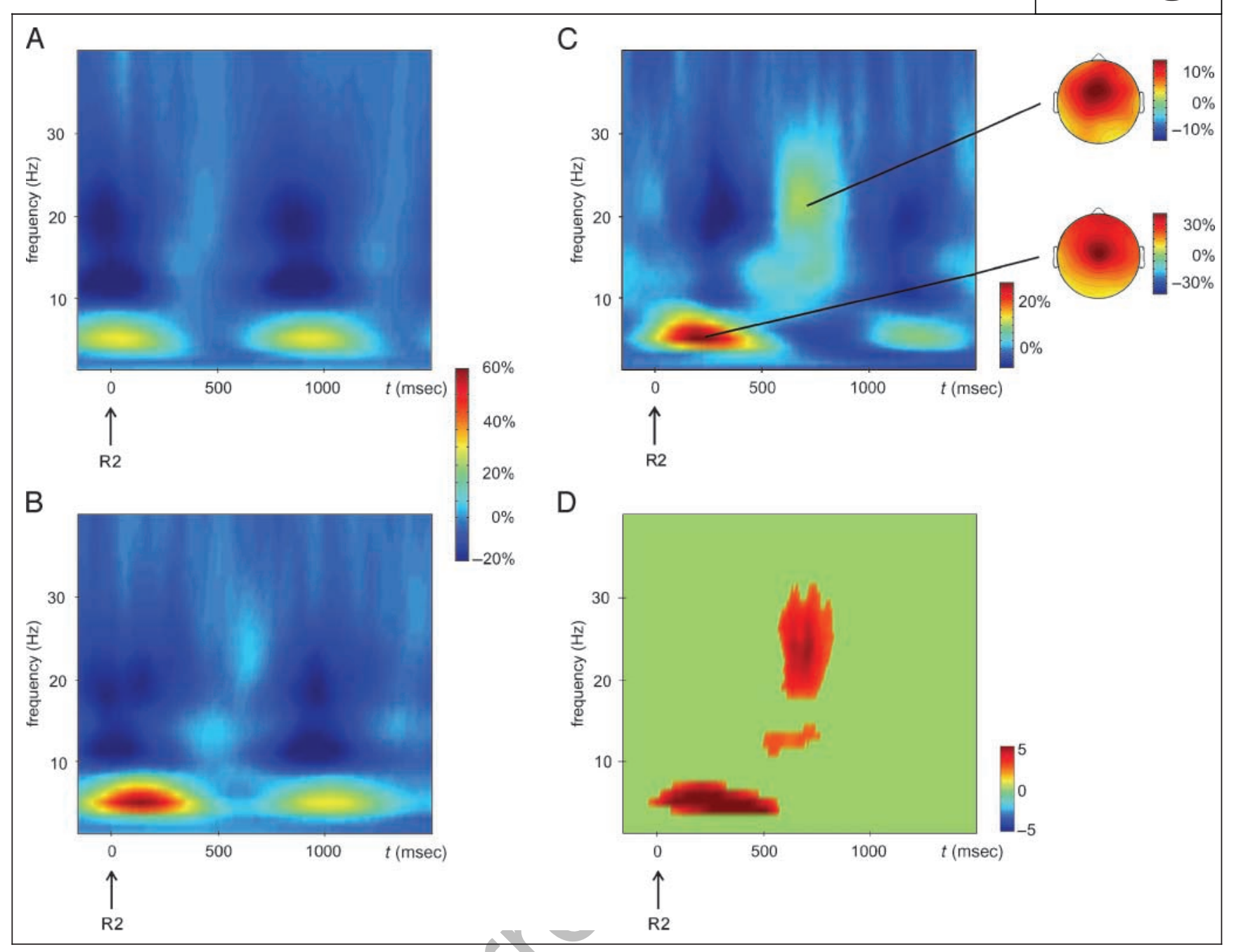

Figure 3. Time-frequency plots for R2 locked data. (A, B) Change of power relative to baseline ( -160 to 0 msec prior to S2) for the correct-correct-correct (CCC) condition (A) and correct-error-correct condition (CEC) (B), from 1 to $40 \mathrm{~Hz}$ at Fz location. Depicted also is the onset of response R2, correct in A and incorrect in B. (C) Differences in the power change between CEC and CCC conditions at Fz electrode. Note the central topography of the theta component and the fronto-central topography of the beta component. (D) Mann-Wilcoxon tests between CEC and CCC conditions at Fz. Only significant values $(p<.05)$ that lasted a minimum of 100 msec are represented.

correlation was found between RT and beta activity $[r=.60, F(1,70)=38.9, p<.001]$.

We performed a similar analysis for the theta band $(0-$ 100 msec after R2) and, again, trials sorted as a function of their response time to R3 showed significant differences $[F(2,46)=3.61, p<.05$; with a significant linear trend, $F(1,23)=4.35, p<.048]$. A further analysis performed for the theta power underlying the Pe component (200-500 msec after R2) did not reveal significant results $[F(2,46)<1]$.

In order to rule out the possibility that the beta oscillatory activity described here is related to the corrective response after the error, we correlated the beta power and the RT of the corrective response. This analysis showed that the beta component was not associated to the production of the corrective response (for slow, medium, and fast corrective responses all $r<.3, p>.1$; when all the responses were pooled, $r=0.2, p>.05$ ].
In sum, our results show a beta oscillatory component that appears at about $600 \mathrm{msec}$ after the commission of an erroneous response and shows a significant correlation with the degree of post-error slowing recorded in the following correct trial after the error. This component was not related to the speed of the corrective response implemented after the error. The correlation between theta and post-error slowing was only significant during the time window of the ERN (0-100 msec), thus corroborating earlier observations implying the ERN component in behavioral adjustments (Debener et al., 2005; Gehring et al., 1993).

\section{Experiment 2}

In the second experiment, we used event-related fMRI (i) to describe the neural network involved in adaptive actions after the commission of an error, and thus, (ii) to 


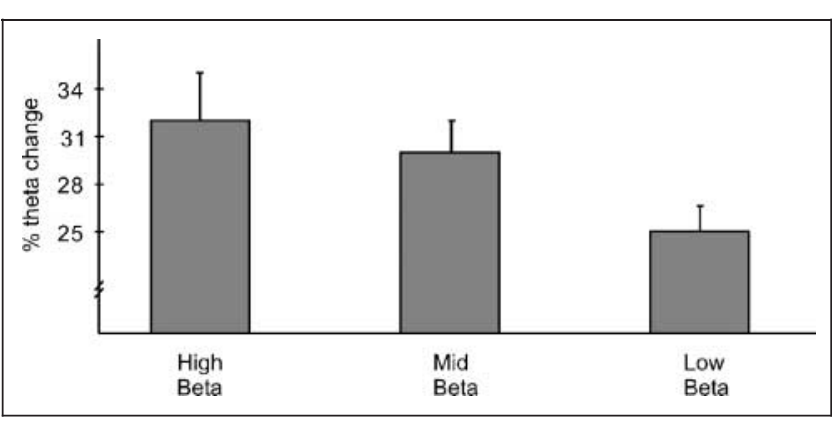

Figure 4. (A) Theta change (0-100 msec after R2, 4-8 Hz) with respect to baseline for trials presenting high (left), medium (middle), and slow (right) beta activity (20-30 Hz, 600-800 msec after R2). Note the linear decrease of theta power with decreasing beta power.

evaluate the possible role of inhibition in post-error adaptations, as has been proposed by the inbibitory account (see above). The participants, therefore, were required to perform the same behavioral task (flanker task) inside the scanner, but on this occasion, we included a variant of the stop-signal task (Logan et al., 1984). With the inclusion of this condition, we delineated the neural network involved in the inhibition of motor commands. Specifically, the inbibitory account predicts overlap between those brain regions related to inhibition (required by the stop signal) and the activation observed in correct trials after the commission of the error. The alternative conflict monitoring account predicts the activation of PFC, in particular, the DLPFC (Egner \& Hirsch, 2005a, 2005b; Kerns et al., 2004; MacDonald, Cohen, Stenger, \& Carter, 2000), as an index of the recruitment of cognitive control mechanisms after high-conflict trials (errors).

\section{Behavioral Results}

The error rate was $12 \pm 3.9 \%$. Again, mean RT for the erroneous responses was significantly faster than for correct responses $[314 \pm 22 \mathrm{msec}$ vs. $382 \pm 22 \mathrm{msec}$, $t(9)=9.98, p<.001]$. The percentage of corrected errors was $94.0 \pm 5.4 \%$ with a mean correction time of $179 \pm 41 \mathrm{msec}$. Additionally, the mean RT for correct responses after an error $(406 \pm 41 \mathrm{msec})$ was significantly slower than that for correct responses after a correct response [373 $\pm 21 \mathrm{msec}, t(9)=4.34, p<.002$ ] The post-error slowing was therefore $32 \mathrm{msec}$. Moreover, the percentage of correct responses after the commission of an error was higher than after a correct trial $[85.9 \pm 2.9 \%$ vs. $84.8 \pm 3.5 \%, t(9)=2.7, p<.02]$.

\section{fMRI Results}

As expected, response errors (when compared to correct responses) gave rise to activations in ACC, extending to the caudal supplementary motor area (SMA). The inferior frontal gyrus (IFG) and the bilateral anterior insular cortex were also activated in this contrast (see Figure 6 and Table 1). This error-related brain network is congruent with results reported elsewhere (e.g., Ullsperger \& von Cramon, 2001; Kiehl, Liddle, \& Hopfinger, 2000). In the stop-signal condition, successfully inhibited stop-signal trials were compared to correct responses. Several regions comprised this inhibitory network: the right middle frontal cortex extending to both the IFG and the DLPFC, the right superior temporal gyrus, left inferior parietal lobe, the precuneus, and the fusiform gyrus (see Figure 6 and Table 1).

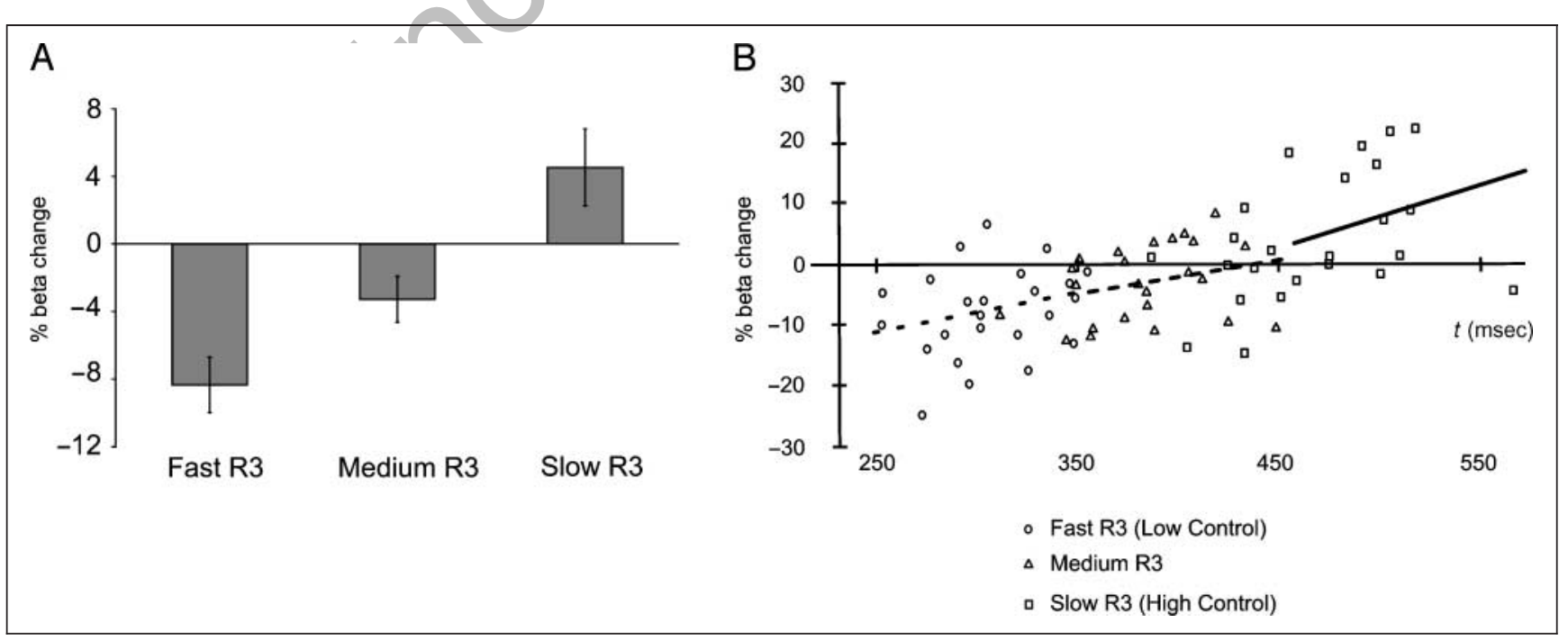

Figure 5. Beta response characteristics. (A) Beta change relative to baseline for trials presenting fast (left), medium (middle), and slow (right) R3 responses (always correct responses). Slow responses, which indicate a larger amount of post-error slowing, showed a larger increase in beta power. (B) Individual change in beta power versus RT for the fast, medium, and slow R3 responses. Note the increase in the beta power when increasing the RT. 


\section{FPO}

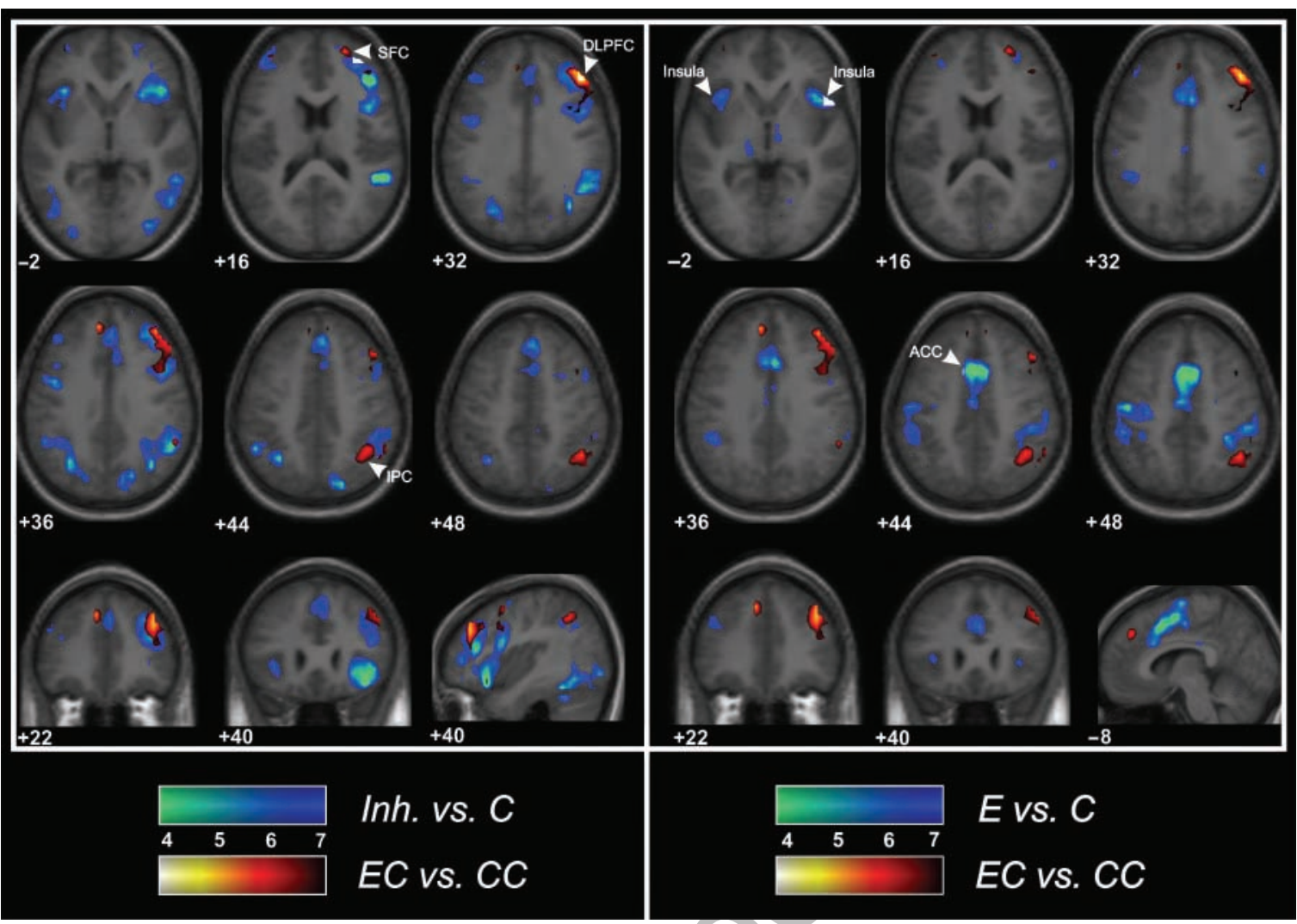

Figure 6. Activations of errors, adaptive actions, and inhibition. (A) Axial views of the group-average activation maps superimposed on a group-averaged structural MRI image in standard stereotactic space ( $t$-score overlays). Correct responses preceded by an erroneous response versus correct responses preceded by a correct response are compared with both error-related network (right) and inhibited-related network (left). Hot patterns refer to brain region associated with EC versus CC activation, whereas winter patterns correspond to the network compared. The right DLPFC was the only region that remained common when the inhibited network was compared to the EC versus CC, whereas no significant overlap between the error-related network and the EC versus CC activation pattern engaged was found. ACC $=$ anterior cingulate cortex; DLPFC $=$ dorsolateral prefrontal cortex; IPC = inferior parietal cortex; SFC = superior frontal cortex.

For the crucial contrast between correct responses preceded by an error (EC sequences) and correct responses preceded by a correct response (CC sequences), three regions were observed to be activated: the right DLPFC, the right superior frontal cortex, and the right inferior parietal cortex (see Figure 6 and Table 1). Notice that the differences present in the contrast EC versus CC cannot be explained by the previous standard error versus correct contrast (see above) due to the fact that no overlap between these regions was encountered (see Figure 6). However, when comparing the EC condition with the inhibitory neural network, both contrasts revealed a significant increase in the right DLPFC, the right superior frontal cortex, and the inferior parietal cortex.

It can be argued that the pattern obtained could be due to a carryover of inhibited trials preceding EC sequences. In order to exclude this possibility, we computed the percentage of inhibited trials that were followed by an EC sequence. EC sequences were preceded by a stop-signal trial in just $5.7 \%$ of the inhibited trials, whereas $8.8 \%$ were followed by a stop-signal trial. Thus, the similarities between EC versus CC and Inh versus $C$ contrasts cannot be explained by the presence of a previous stop trial.

Important for our aim to distinguish inhibitory and conflict monitoring accounts is the question whether the regions found in the crucial EC versus CC contrast is more similar to those activated in the error versus correct contrast ("error-related network") or the inhibited versus correct contrast ("inhibitory network"). To this end, we first identified the regions that were activated in the error-related network and the inhibitory network by thresholding the corresponding statistical map at $p<.001$ (uncorrected, cluster spatial extent exceeded 20 voxels). Second, we masked the EC versus CC contrast with either the error-related or the inhibitory network. The right DLPFC was the only region that showed significant overlap ( $p<.001$, uncorrected) between the EC versus CC activation pattern and the inhibitory network. By contrast, no common regions were observed between the error-related network and the EC versus CC contrast. This result suggests partial overlap between the inhibitory network and the regions activated by the correct responses preceded by an error. 
Table 1. Brain Regions Showing Changes in Activity Comparing Erroneous vs. Correct Responses (E-C), Inhibited Trials vs. Correct Responses (I-C), and EC vs. CC Responses (EC-CC)

\begin{tabular}{|c|c|c|c|c|c|}
\hline \multirow[b]{2}{*}{ Brain Region } & \multirow[b]{2}{*}{$\sim B A$} & \multicolumn{3}{|c|}{$\begin{array}{l}\text { Stereotactic } \\
\text { Coordinates }\end{array}$} & \multirow[b]{2}{*}{ t Peak } \\
\hline & & $x$ & $y$ & $z$ & \\
\hline \multicolumn{6}{|l|}{$E-C$} \\
\hline \multirow[t]{2}{*}{ SMA/ACC } & 32 & 0 & 12 & 48 & 10.33 \\
\hline & 6 & -4 & 0 & 52 & 9.31 \\
\hline L Primary motor & 4 & -36 & -28 & 60 & 8.47 \\
\hline R IPG & 40 & 48 & -36 & 52 & 5.93 \\
\hline $\begin{array}{l}\text { R IFG/anterior } \\
\text { insula }\end{array}$ & & 44 & 16 & -8 & 7.15 \\
\hline $\begin{array}{l}\text { L IFG/anterior } \\
\text { insula }\end{array}$ & & -40 & 16 & 4 & 6.47 \\
\hline \multicolumn{6}{|l|}{$I-C$} \\
\hline R IFG & 47 & 40 & 24 & -16 & 12.20 \\
\hline R DLPFC & 46 & 44 & 32 & 16 & 9.35 \\
\hline \multirow[t]{2}{*}{ R IPG/R STG } & $40 / 22$ & 48 & -44 & 20 & 10.67 \\
\hline & 22 & 56 & -44 & 16 & 9.42 \\
\hline Fusiform gyrus & 37 & 44 & -52 & -16 & 8.12 \\
\hline Precuneus & 7 & 12 & -76 & 40 & 7.77 \\
\hline SFG & 6 & 20 & 4 & 64 & 7.76 \\
\hline L IPG & 40 & -32 & -56 & & 7.46 \\
\hline $\begin{array}{l}\text { Occipital gyrus/ } \\
\text { Fusiform gyrus }\end{array}$ & 19 & -44 & & -12 & 7.06 \\
\hline MFG & 8 & & 32 & 44 & 5.83 \\
\hline \multicolumn{6}{|l|}{$E C-C C$} \\
\hline R DLPFC & 46 & 44 & 36 & 32 & 6.41 \\
\hline L MFC & 9 & -8 & 44 & 36 & 6.03 \\
\hline R SFG & 10 & 24 & 56 & 16 & 5.28 \\
\hline R IPG & 40 & 44 & -56 & 48 & 5.31 \\
\hline
\end{tabular}

MNI coordinates and $t$ value for the peak location are given for each cluster showing significant differences $(p<.001,20$ voxels spatial extent) $\mathrm{BA}=$ approximate Brodmann's area; $\mathrm{L}=$ left hemisphere; $\mathrm{R}=$ right hemisphere; $\mathrm{SMA}=$ supplementary motor area; $\mathrm{ACC}=$ anterior cingulate cortex; IFG $=$ inferior frontal gyrus; DLPFC $=$ dorsolateral prefrontal cortex; IPG = inferior parietal gyrus; STG = superior temporal gyrus; $\mathrm{MFG}=$ medial frontal gyrus; $\mathrm{SFG}=$ superior frontal gyrus; $\mathrm{MFC}=$ middle frontal cortex.

As the number of participants in the present study was limited, we investigated whether the pattern of activation obtained in the right DLPFC was consistent across participants. After defining an ROI located in the significant right DLPFC cluster, all participants presented consistent activation patterns, recording higher values for the peak of the reconstructed hemodynamic response in the EC sequences and lower values in CC sequences [Figure 7; $0.58 \pm 0.42 \%$ signal change vs. $0.14 \pm$ $0.1 \%$ signal change, $t(9)=3.72, p<.005]$. This result corroborates the involvement of this region in the posterror slowing phenomenon.

Finally, we needed to reject the possibility that the right DLPFC might reflect conflict-related activations related to stimulus-response compatibility (incongruency). Typically, erroneous responses occur more often for incongruent stimuli, whereas correct responses comprise congruent and incongruent trials. In other words, error-correct sequences might reflect underlying conflict-related activations, that is, high conflict in incongruent trials might lead to the greater cognitive control shown in the following trial. In order to test this hypothesis, a new statistical analysis was performed including stimulus-response compatibility sequences as factors in the design matrix. However, when trials preceded by incongruent trials were compared to the trials preceded by a congruent one, no significant activation was found in the right DLPC. This result suggests that this region was not related to the conflict adaptation mechanism instigated by the presence of incongruent trials in the previous trial.

\section{Experiment 3}

The fMRI results clearly suggested the involvement of brain regions related to motor inhibition in post-error adaptation. We thus wanted to investigate whether the oscillatory components in the beta and theta range found in Experiment 1 as correlates of post-error slowing could be linked to the inhibition of motor commands. With this aim in mind, we designed an EEG experiment that, similar to the fMRI study, combined a standard flanker task with a stop-signal condition. Stop signals occurred in $16.7 \%$ of the trials and stop-signal delay was determined using an ideal staircase tracking algorithm in order to yield enough successfully inhibited and noninhibited stop trials ( 50\% in each condition) (Band \& van Boxtel, 1999). We predicted the presence of the beta component in successfully inhibited trials when compared to noninhibited trials and correct responses. Responses to the standard flanker stimuli will not be considered.

\section{Behavioral Results}

The mean percentage of noninhibited trials was $51.3 \pm$ $6.1 \%$. In the noninhibited trials, the stop signal appeared with a mean delay of $152 \pm 8 \mathrm{msec}$ after the presentation of the imperative stimuli, whereas the stop-signal de- 


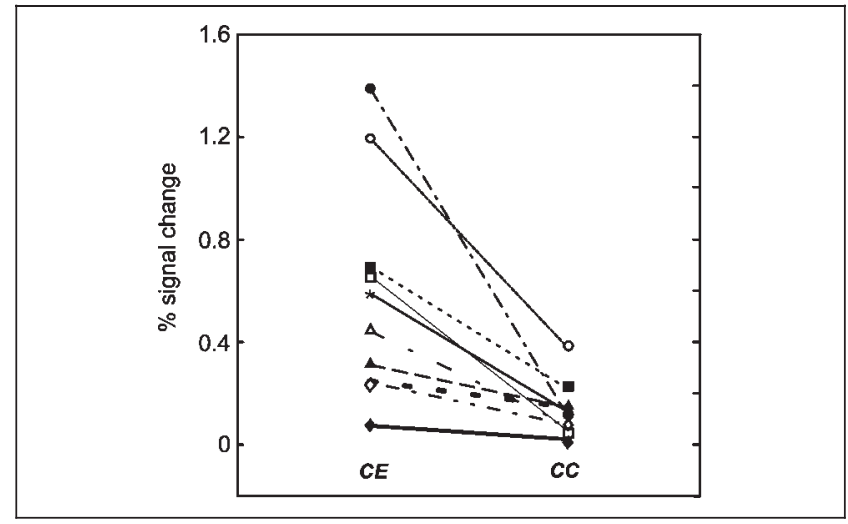

Figure 7. Peak activation of the reconstructed hemodynamic response in the selected right DLPFC. ROI is compared within-subjects between the EC and CC conditions, showing higher values for the EC sequences and lower ones in CC sequences for all participants.

lay in the inhibited trials appeared at about $113 \pm 35 \mathrm{msec}$ $[t(23)=5.74, p<.001]$. In noninhibited trials, the mean RT of the responses was $221 \pm 104$ msec after the stop signal.

\section{Time-frequency Analysis}

Figure 8A shows the difference of time-frequency analysis between inhibited and noninhibited trials locked to the appearance of the stop stimulus. A comparison of theta band power showed a greater increase $(4-6 \mathrm{~Hz}$, 300-500 msec after stimuli) for noninhibited trials compared to inhibited trials $[F(1,23)=24.2, p<.001$ between both conditions; Condition $\times$ Electrode: $F(2$, $46)=22.5, p<.001$; see Figure 8A]. This increase in the theta component is most probably related to the commission of an error. And, as such, is associated with an ERN component for noninhibited trials in the timedomain averages (not shown). On the other hand, inhibited trials presented greater power at the beta band (20-30 Hz, 350-550 msec) when compared to noninhibited trials $[F(1,23)=24.2, p<.001]$.

The beta response associated with inhibition also appeared when we compared successfully inhibited trials and correct responses in standard trials matched in RT to the noninhibited responses. The beta increase in the inhibited trials compared to that in the correct responses appeared in the time range 450-550 msec after the standard flanker stimulus [see Figure 8B; Electrode $\times$ Condition $F(2,46)=7.4, p<.005]$, with a frontal distribution $[t(23)=3.36, p<.005$ for $\mathrm{Fz}$, but not significant for $\mathrm{Cz}$ and $\mathrm{Pz}]$.

\section{DISCUSSION}

This study investigates cognitive control processes triggered after the commission of an error. Following action errors, subjects may (a) correct the error by producing a corrective response and (b) adapt their response speed and accuracy in subsequent trials in order to avoid additional errors. Both behavioral adaptation effects were present in our experiments. Electrophysiologically, we identified oscillatory beta activity in the EEG that correlates with the increase in cognitive control reflected by post-error slowing. The results of Experiment 3, showing a greater beta response for strop trials requiring inhibition of a motor response, further suggest that this beta component is related to inhibitory processes. In addition, the event-related fMRI experiment implies that cognitive control is implemented through the activity of an inhibitory neural network.

Correct trials following an error compared to correct trials after a correct response showed activations in the

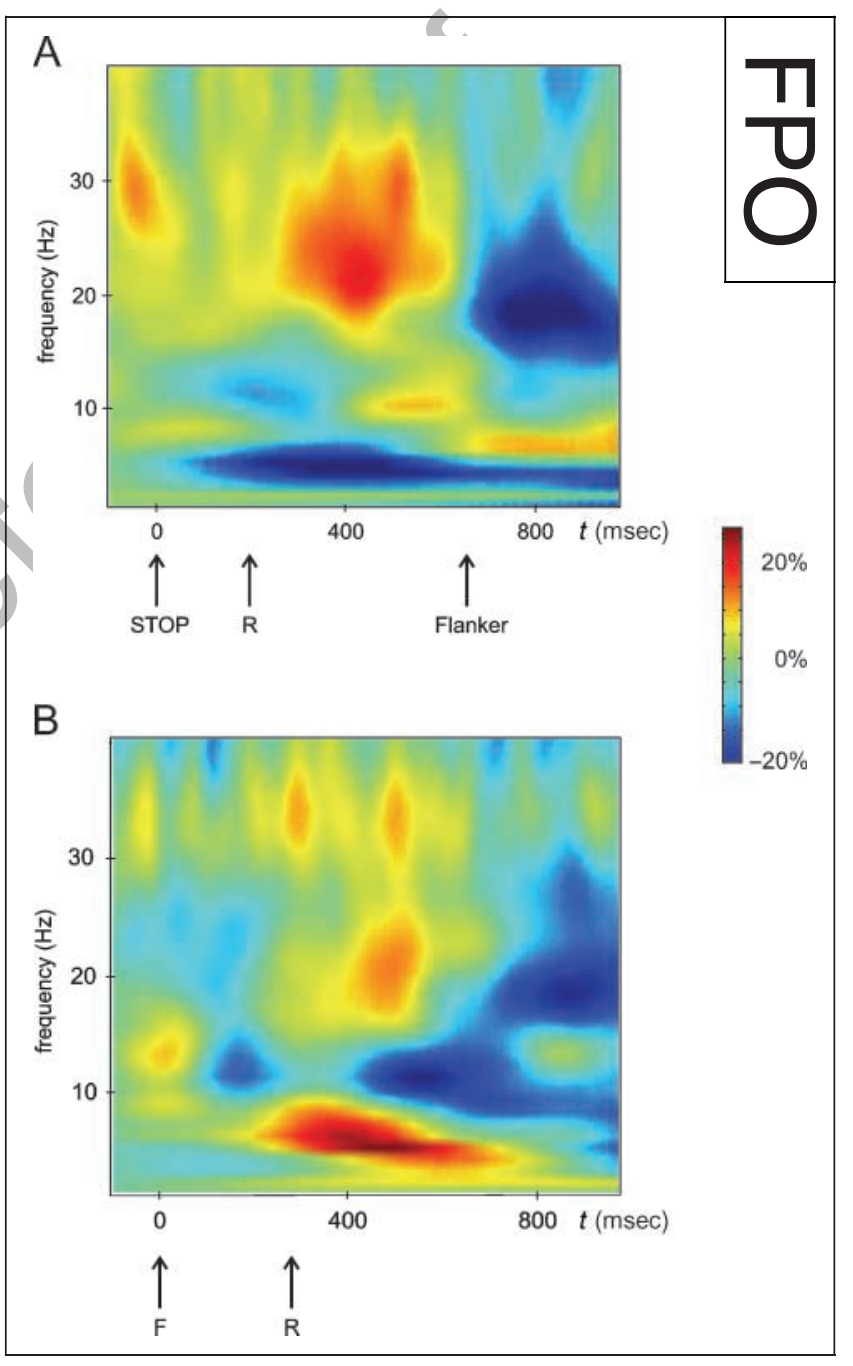

Figure 8. (A) Differences in the power change between inhibited and noninhibited conditions at the Fz electrode. STOP arrow indicates the incoming of stop stimulus, $\mathrm{R}$ arrow indicates the incoming of the response in noninhibited condition, and the flanker arrow indicates the incoming of the new flanker stimulus. Note the difference in the beta power (20-30 Hz) between 350 and 550 msec. (B) Differences in the power change between inhibited and correct response conditions at the $\mathrm{Fz}$ electrode. Inhibited and correct responses are matched in RT to noninhibited responses. Note the difference in the beta power (20-30 Hz) between 450 and $550 \mathrm{msec}$. 
right DLPFC extending to the superior frontal gyrus and in the right inferior parietal cortex (see Figure 6 and Table 1). These activations differed markedly from those observed in the contrast between erroneous and correct responses, that is, the "error-related network," while they strongly resembled those seen when participants had to inhibit a response after the presentation of a stop signal (see Table 1). Hence, the present results suggest that the brain areas associated with the detection of an error can be dissociated from those involved in instigating the control processes needed for post-error adaptive actions. Moreover, the coincidence between areas involved in the adaptive actions after slips and those involved in inhibition of already initiated actions suggest that the increased cognitive control observed after an error (post-error slowing) is most probably implemented through an inhibitory network encompassing the DLPFC and the inferior parietal cortex. The right DLPFC has been clearly associated with inhibition using a lesion approach in monkeys (Mishkin, 1964). In addition, the right DLPFC and the inferior parietal cortex have been found to be activated in go/no-go and stop-signal experiments both in humans (de Zubicaray, Zelaya, Andrew, Williams, \& Bullmore, 2000; Garavan, Ross, \& Stein, 1999) and nonhuman primates (Sakagami et al., 2001; Sasaki, Gemba, \& Tsujimoto, 1989), and some studies have proposed it as one of the main locus for inhibition (see meta-analysis by Simmonds, Pekar, \& Mostofsky, in press; Wager et al., 2005; but see Aron, Robbins, \& Poldrack, 2004 for a proposal of the right IFG as an inhibitory locus). Hence, the present results support the inhibitory account (Ridderinkhof, 2002).

Critically, the interpretation of the activation patterns to post-error trials is dependent on the functions subserved by the DLPFC. In addition to its role in inhibition, the DLPFC plays an important role in cognitive control (see Johnston \& Everling, 2006; MacDonald et al., 2000). Thus, the present results would also be compatible with the conflict monitoring account (Botvinick et al., 2001) that proposes that the increased conflict resulting from the error might trigger cognitive control mechanisms instantiated in PFC. In this model, ACC subserves for conflict monitoring (Kerns et al., 2004) as the prerequisite for subsequent remedial actions (Botvinick, Cohen, \& Carter, 2004; Kerns et al., 2004; Botvinick et al., 2001; see, however, Nakamura, Roesch, \& Olson, 2005 for results that question the role of ACC in conflict monitoring). After the detection of the conflict in ACC, the DLPFC enhances cognitive control in order to reduce the conflict. Finally, this leads to a bias of information processing in other areas of the brain in charge of perceptual or motor processing (Egner \& Hirsch, 2005b). In this regard, the present data add to the existing literature on the interplay between ACC and PFC in monitoring and controlling behavior.

Given the two alternative accounts, inhibition and conflict monitoring, the question arises how the DLPFC would support these processes. The DLPFC has reciprocal connections with ACC (Paus, Castro-Alamancos, \& Petrides, 2001), the inferior parietal cortex, the SMA, the pre-SMA, and the premotor cortex (Ridderinkhof, van den Wildenberg, Segalowitz, \& Carter, 2004; Paus et al., 2001; Koski \& Paus, 2000; Petrides \& Pandya, 1999). With regard to inhibition, it has been proposed that the right PFC could exert his function by suppressing basal ganglia output to the motor regions via the subthalamic nucleus (Aron \& Poldrack, 2006; Aron et al., 2004). Thus, the activation of the DLPFC in the present dataset could be interpreted as the correlate of a top-down control signal that increases the amount of inhibition after an error, and thus, produces the post-error slowing effect.

Alternatively, in the context of the conflict monitoring account, the DLPFC top-down signal regulates the amount of activation at the motor level. A trial-by-trial response priming mechanism maintains the system in different motor readiness states that depend on the degree of conflict experienced in the previous trial. In computational implementations (Brown, Reynolds, \& Braver, 2007; Jones, Cho, Nystrom, Cohen, \& Braver, 2002), cognitive control influences the motor-response level via a tonic arousal signal that affects the degree of activation of the motor channel. The decrease of this signal produces the slowing of the next trial of the sequence. As is apparent from this discussion, the distinction between inhibitory and conflict monitoring accounts on the neural level is not trivial and awaits further investigation. Moreover, a recent model proposes different conflict-control loops, each associated with specific forms of compensatory adjustments as they become necessary, for example, after a task switch, on incongruent trials or, as our study, erroneous responses (Brown et al., 2007). Interestingly, the computational simulation performed in Brown et al. (2007), including only a single conflict-control mechanism, was unable to account for the corresponding empirical observations. These results further stress the importance of dissecting these types of conflict-control loops and their implications in behavioral adjustments.

As the simulations of Brown et al. (2007) suggest, it is important to rule out that the activation observed in the right PFC was due to a larger percentage of incongruent trials in error responses, as the conflict-control mechanisms on incongruent trials should be different from those underlying post-error slowing.

We also investigated how behavioral adaptation processes are reflected by the electrical activity of the brain. The principal result is an increase in the fronto-central beta activity $(20-30 \mathrm{~Hz})$ at $600-800 \mathrm{msec}$ after the commission of an error. This component is correlated to the power of the theta activity underlying the ERN component. In addition, this oscillatory activity is related to post-error slowing but not to the speed of correction. Moreover, the corrective command occurs approximately $200 \mathrm{msec}$ after the commission of the error, and thus, 
400-500 msec before the appearance of the fronto-central beta component. Its scalp distribution also argues against the possibility that it is an instance of motor-related beta, which has been described overlying sensorimotor areas (Neuper \& Pfurtscheller, 2001).

Importantly, the current datasets allow to relate the various electrophysiological and neuroimaging markers of error processing to the behavioral effects of action monitoring, in particular, post-error slowing. To establish a bridge between these levels of observation is a key task in action monitoring research. In this regard, it is important to assess the relation between the brain regions activated in the fMRI study (EC/CC contrast) and the EEG betaresponse, as both are related to adaptive actions following an error. An increase in EEG beta activity has been found in a number of divergent tasks involving different cognitive processes [i.e., semantic memory recall (Slotnick, Moo, Kraut, Lesser, \& Hart, 2002) and working memory (Palva, Palva, \& Kaila, 2005)]. Theoretical studies have proposed that oscillatory beta activity might be a good candidate for connecting distant brain structures (Kopell, Ermentrout, Whittington, \& Traub, 2000). This has been corroborated by simultaneous measurements from depth and surface electrodes (Steriade, 2006; Fogelson et al., 2005), which show that beta-activity mediates coupling within the functional loops between the basal ganglia, thalamus, and different cortical areas. The functional role of beta activity in the present study might thus be the integration of the activity in the neural network involved in post-error processing, as described in the fMRI study (lateral frontal cortex, medial frontal cortex, and inferior parietal cortex). Interestingly, beta activity has also been related to inhibition in go/no-go (Alegre et al., 2004) and motor (Neuper \& Pfurtscheller, 2001) studies.

In summary, our results suggest that the adaptive actions after the commission of an error are produced by a neural circuit involving the right DLPFC and the inferior parietal cortex. Moreover, this activation is related to an increase in EEG beta activity $600-800$ msec after the error, which is correlated to post-error slowing and the theta response associated with ERN. The coincidence of the circuit with the areas activated in the inhibition of responses suggests an inhibitory mechanism to be involved in the increase of cautiousness observed after the commission of an error.

\section{Acknowledgments}

We thank the reviewers of the present manuscript for their helpful comments. This research was supported by research grants of Spanish Government (SEJ2005-06067/PSIC to A. R. F.), Generalitat de Catalunya (SGR2005-00831) and the Volkswagenstiftung to A. R. F. and T. F. M. T. F. M. is also supported by the DFG and the BMBF. J. M. P. is a fellow of the Alexander-vonHumboldt-Foundation.

Reprint requests should be sent to Josep Marco-Pallarés, Otto von Guericke University, Department of Neuropsychology, Universitätsplatz, Gebäude 24, Postfach 4120, 39106 Magdeburg, Germany, or via e-mail: josepmarco@hotmail.com.

\section{REFERENCES}

Alegre, M., Gurtubay, I. G., Labarga, A., Iriarte, J., Valencia, M., \& Artieda, J. (2004). Frontal and central oscillatory changes related to different aspects of the motor process: A study in go/no-go paradigms. Experimental Brain Research, 159, 14-22.

Aron, A. R., \& Poldrack, R. A. (2006). Cortical and subcortical contributions to stop signal response inhibition: Role of the subthalamic nucleus. Journal of Neuroscience, 26, 2424-2433.

Aron, A. R., Robbins, T. W., \& Poldrack, R. A. (2004). Inhibition and the right inferior frontal cortex. Trends in Cognitive Sciences, 8, 170-177.

Ashburner, J., \& Friston, K. J. (1999). Nonlinear spatial normalization using basis functions. Human Brain Mapping, 7, 254-266.

Band, G. P., \& van Boxtel, G. J. (1999). Inhibitory motor control in stop paradigms: Review and reinterpretation of neural mechanisms. Acta Psychologica (Amsterdam), 101, 179-211.

Botvinick, M. M., Braver, T. S., Barch, D. M., Carter, C. S., \& Cohen, J. D. (2001). Conflict monitoring and cognitive control. Psychological Review, 108, 624-652.

Botvinick, M. M., Cohen, J. D., \& Carter, C. S. (2004). Conflict monitoring and anterior cingulate cortex: An update. Trends in Cognitive Sciences, 8, 539-546.

Brown, J. W., Reynolds, J. R., \& Braver, T. S. (2007). A computational model of fractionated conflict-control mechanisms in task-switching. Cognitive Psychology, 55, 37-85.

Burns, J. T. (1971). Error-induced inhibition in a serial reaction time task. Journal of Experimental Psychology, 90, 141-148.

Cocosco, C. A., Kollokian, V., Kwan, R. K. S., \& Evan, A. C. (1997). BrainWeb: Online interface to a 3-D MRI simulated brain database. Neuroimage, 5, S425.

de Zubicaray, G. I., Zelaya, F. O., Andrew, C., Williams, S. C., \& Bullmore, E. T. (2000). Cerebral regions associated with verbal response initiation, suppression and strategy use. Neuropsychologia, 38, 1292-1304.

Debener, S., Ullsperger, M., Siegel, M., Fiehler, K., von Cramon, D. Y., \& Engel, A. K. (2005). Trial-by-trial coupling of concurrent electroencephalogram and functional magnetic resonance imaging identifies the dynamics of performance monitoring. Journal of Neuroscience, 25, 11730-11737.

Egner, T., \& Hirsch, J. (2005a). Cognitive control mechanisms resolve conflict through cortical amplification of taskrelevant information. Nature Neuroscience, 8, 1784-1790.

Egner, T., \& Hirsch, J. (2005b). The neural correlates and functional integration of cognitive control in a Stroop task. Neuroimage, 24, 539-547.

Falkenstein, M., Hohnsbein, J., Hoormann, J., \& Blanke, L. (1990). Effects of errors in choice reaction task on the ERP under focused and divided attention. In C. H. M. Brunia, A. W. K. Gaillard, \& A. Kok (Eds.), Psychophysiological brain research (pp. 192-195). Tilburg, The Netherlands: University Press.

Fogelson, N., Pogosyan, A., Kuhn, A. A., Kupsch, A., van Bruggen, G., Speelman, H., et al. (2005). Reciprocal interactions between oscillatory activities of different frequencies in the subthalamic region of patients with Parkinson's disease. European Journal of Neuroscience, 22, 257-266.

Friston, K. J., Fletcher, P., Josephs, O., Holmes, A., Rugg, M. D., \& Turner, R. (1998). Event-related fMRI: Characterizing differential responses. Neuroimage, 7, 30-40.

Friston, K. J., Williams, S., Howard, R., Frackowiak, R. S., \& Turner, R. (1996). Movement-related effects in fMRI timeseries. Magnetic Resonance in Medicine, 35, 346-355. 
Garavan, H., Ross, T. J., Murphy, K., Roche, R. A., \& Stein, E. A. (2002). Dissociable executive functions in the dynamic control of behavior: Inhibition, error detection, and correction. Neuroimage, 17, 1820-1829.

Garavan, H., Ross, T. J., \& Stein, E. A. (1999). Right hemispheric dominance of inhibitory control: An event-related functional MRI study. Proceedings of the National Academy of Sciences, U.S.A., 96, 8301-8306.

Gehring, W. J., \& Fencsik, D. E. (2001). Functions of the medial frontal cortex in the processing of conflict and errors. Journal of Neuroscience, 21, 9430-9437.

Gehring, W. J., Goss, B., Coles, M. G. H., Meyer, D. E., \& Donchin, E. (1993). A neural system for error-detection and compensation. Psychological Science, 4, 385-390.

Gehring, W. J., \& Willoughby, A. R. (2004). Are all medial frontal negativities created equal? Toward a richer empirical basis for theories of action monitoring. In M. Ullsperger \& M. Falkenstein (Eds.), Errors, conflicts, and the brain. Current opinions on performance monitoring (pp. 14-20). Leipzig: Max Planck Institute of Cognitive Science.

Gratton, G., Coles, M. G., \& Donchin, E. (1992). Optimizing the use of information: Strategic control of activation of responses. Journal of Experimental Psychology: General, 121, 480-506.

Johnston, K., \& Everling, S. (2006). Monkey dorsolateral prefrontal cortex sends task-selective signals directly to the superior colliculus. Journal of Neuroscience, 26, 12471-12478.

Jones, A. D., Cho, R. Y., Nystrom, L. E., Cohen, J. D., \& Braver, T. S. (2002). A computational model of anterior cingulate function in speeded response tasks: Effects of frequency, sequence, and conflict. Cognitive, Affective \& Behavioral Neuroscience, 2, 300-317.

Kerns, J. G., Cohen, J. D., MacDonald, A. W., Cho, R. Y., Stenger, V. A., \& Carter, C. S. (2004). Anterior cingulate conflict monitoring and adjustments in control. Science, 303, 1023-1026.

Kiehl, K. A., Liddle, P. F., \& Hopfinger, J. B. (2000). Error processing and the rostral anterior cingulate: An eventrelated fMRI study. Psychophysiology, 37, 216-223.

Klein, T. A., Endrass, T., Kathmann, N., Neumann, J., von Cramon, D. Y., \& Ullsperger, M. (2007). Neural correlates of error awareness. Neuroimage, 34, 1774-1781.

Kopell, N., Ermentrout, G. B., Whittington, M. A., \& Traub, R. D. (2000). Gamma rhythms and beta rhythms have different synchronization properties. Proceedings of the National Academy of Sciences, U.S.A., 97, 1867-1872.

Koski, L., \& Paus, T. (2000). Functional connectivity of the anterior cingulate cortex within the human frontal lobe: A brain-mapping meta-analysis. Experimental Brain Research, $133,55-65$.

Laming, D. (1979). Choice reaction performance following an error. Acta Psychologica, 43, 199-224.

Logan, G. D., Cowan, W. B., \& Davis, K. A. (1984). On the ability to inhibit simple and choice reaction time responses: A model and a method. Journal of Experimental Psychology: Human Perception and Performance, 10, 276-291.

Luu, P., Tucker, D. M., Derryberry, D., Reed, M., \& Poulsen, C. (2003). Electrophysiological responses to errors and feedback in the process of action regulation. Psychological Science, 14, 47-53.

MacDonald, A. W., Cohen, J. D., Stenger, V. A., \& Carter, C. S. (2000). Dissociating the role of the dorsolateral prefrontal and anterior cingulate cortex in cognitive control. Science, 288, 1835-1838.

Mayr, U., Awh, E., \& Laurey, P. (2003). Conflict adaptation effects in the absence of executive control. Nature Neuroscience, 6, 450-452.
Mishkin, M. (1964). Perseveration of central sets after frontal lesions in monkeys. In J. M. Warren \& K. Akert (Eds.), The frontal granular cortex and behavior (pp. 219-241). New York: McGraw-Hill.

Nakamura, K., Roesch, M. R., \& Olson, C. R. (2005). Neuronal activity in macaque SEF and ACC during performance of tasks involving conflict. Journal of Neurophysiology, 93, 884-908.

Neuper, C., \& Pfurtscheller, G. (2001). Event-related dynamics of cortical rhythms: Frequency-specific features and functional correlates. International Journal of Psychophysiology, 43, 41-58.

Nieuwenhuis, S., Ridderinkhof, K. R., Blom, J., Band, G. P., \& Kok, A. (2001). Error-related brain potentials are differentially related to awareness of response errors: Evidence from an antisaccade task. Psychophysiology, 38, 752-760.

Palva, J. M., Palva, S., \& Kaila, K. (2005). Phase synchrony among neuronal oscillations in the human cortex. Journal of Neuroscience, 25, 3962-3972.

Paus, T., Castro-Alamancos, M. A., \& Petrides, M. (2001). Cortico-cortical connectivity of the human mid-dorsolateral frontal cortex and its modulation by repetitive transcranial magnetic stimulation. European Journal of Neuroscience, 14, 1405-1411.

Petrides, M., \& Pandya, D. N. (1999). Dorsolateral prefrontal cortex: Comparative cytoarchitectonic analysis in the human and the macaque brain and corticocortical connection patterns. European Journal of Neuroscience, 11, 1011-1036.

Picton, T. W., Bentin, S., Berg, P., Donchin, E., Hillyard, S. A., Johnson, R., et al. (2000). Guidelines for using human eventrelated potentials to study cognition: Recording standards and publication criteria. Psychophysiology, 37, 127-152.

Rabbitt, P. (1990). Age, IQ and awareness, and recall of errors. Ergonomics, 33, 1291-1305.

Rabbitt, P. (2002). Consciousness is slower than you think. Quarterly Journal of Experimental Psychology A, 55, 1081-1092.

Rabbitt, P. M. (1966). Errors and error correction in choiceresponse tasks. Journal of Experimental Psychology, 71, 264-272.

Rabbitt, P. M. (1968). Three kinds of error-signalling responses in a serial choice task. Quarterly Journal of Experimental Psychology, 20, 179-188.

Riba, J., Rodriguez-Fornells, A., Morte, A., Munte, T. F., \& Barbanoj, M. J. (2005). Noradrenergic stimulation enhances human action monitoring. Journal of Neuroscience, 25, 4370-4374.

Riba, J., Rodriguez-Fornells, A., Munte, T. F., \& Barbanoj, M. J. (2005). A neurophysiological study of the detrimental effects of alprazolam on human action monitoring. Brain Research, Cognitive Brain Research, 25, 554-565.

Ridderinkhof, K. R. (2002). Micro- and macro-adjustments of task set: Activation and suppression in conflict tasks. Psychological Research, 66, 312-323.

Ridderinkhof, K. R., van den Wildenberg, W. P., Segalowitz, S. J., \& Carter, C. S. (2004). Neurocognitive mechanisms of cognitive control: The role of prefrontal cortex in action selection, response inhibition, performance monitoring, and reward-based learning. Brain and Cognition, 56, 129-140.

Rodriguez-Fornells, A., Kurzbuch, A. R., \& Munte, T. F. (2002). Time course of error detection and correction in humans: Neurophysiological evidence. Journal of Neuroscience, 22, 9990-9996.

Sakagami, M., Tsutsui, K., Lauwereyns, J., Koizumi, M., Kobayashi, S., \& Hikosaka, O. (2001). A code for behavioral 
inhibition on the basis of color, but not motion, in ventrolateral prefrontal cortex of macaque monkey. Journal of Neuroscience, 21, 4801-4808.

Sasaki, K., Gemba, H., \& Tsujimoto, T. (1989). Suppression of visually initiated hand movement by stimulation of the prefrontal cortex in the monkey. Brain Research, 495, 100-107.

Simmonds, D. J., Pekar, J. J., \& Mostofsky, S. H. (in press). Meta-analysis of Go/No-go tasks demonstrating that fMRI activation associated with response inhibition is taskdependent. Neuropsychologia.

Slotnick, S. D., Moo, L. R., Kraut, M. A., Lesser, R. P., \& Hart, J., Jr. (2002). Interactions between thalamic and cortical rhythms during semantic memory recall in human.

Proceedings of the National Academy of Sciences, U.S.A., 99, 6440-6443.

Steriade, M. (2006). Grouping of brain rhythms in corticothalamic systems. Neuroscience, 137, 1087-1106.

Stuphorn, V., Taylor, T. L., \& Schall, J. D. (2000). Performance monitoring by the supplementary eye field. Nature, 408 , 857-860.
Tallon-Baudry, C., Bertrand, O., Delpuech, C., \& Permier, J. (1997). Oscillatory gamma-band (30-70 Hz) activity induced by a visual search task in humans. Journal of Neuroscience, $17,722-734$

Ullsperger, M., \& von Cramon, D. Y. (2001). Subprocesses of performance monitoring: A dissociation of error processing and response competition revealed by event-related fMRI and ERPs. Neuroimage, 14, 1387-1401.

Wager, T. D., Sylvester, C. Y. C., Lacey, S. C., Nee, D. E., Franklin, M., \& Jonides, J. (2005). Common and unique components of response inhibition revealed by fMRI. Neuroimage, 27, 323-340.

Wendt, M., Heldmann, M., Munte, T. F., \& Kluwe, R. H. (2007). Disentangling sequential effects of stimulus- and responserelated conflict and stimulus-response repetition using brain potentials. Journal of Cognitive Neuroscience, 19, 1104-1112.

Worsley, K. J., \& Friston, K. J. (1995). Analysis of fMRI timeseries revisited-again. Neuroimage, 2, 173-181.

Yeung, N., Botvinick, M. M., \& Cohen, J. D. (2004). The neural basis of error detection: Conflict monitoring and the errorrelated negativity. Psychological Review, 111, 931-959. 